\title{
Reviewer's comment concerning "Influence of a variation in the position of the arms on the sagittal connection of the gravity line with the spinal structures" (by J. Legaye and G. Duval-Beaupere, Eur Spine J; 2017: doi:10.1007/ s00586-017-4961-6)
}

\author{
Pedro Berjano ${ }^{1}$
}

Received: 2 October 2017 / Accepted: 3 October 2017 / Published online: 13 October 2017

(c) Springer-Verlag GmbH Germany 2017

Any reviewer would feel honored and overwhelmed by the endeavor of reviewing and commenting on an article from Jean Legaye and Ginette Duval-Beaupère. For the last 20 (the former) and 40 (the latter) years, they have made game changing contributions to our spinal knowledge, including, but not limited to, their description of pelvic incidence and its relationship to the spinal sagittal profile [1].

The research question of this article (how to compute the influence of the arms' position on the displacement of the center of gravity of the standing human body) was triggered by discrepancies found among different studies (references 9-14 in this article). In fact, those studies showed conflicting results regarding the position of the gravity line in standing humans that the authors (JL and GD-B) interpret as being the effect of different arm positions.

In this article, data from a previous investigation [2] have been re-analyzed and interpreted to provide a method that allows to estimate the changes that different positions of the arms induce in the position of the standing body gravity line. The authors have proposed a mathematical calculation that would explain the findings of the cited articles.

Some limitations are present in the study.

First, the data used to make the calculations are historical and might not be reproducible due to concerns regarding exposure of volunteers to radiation (the estimation of the center of mass of the body slices was made exposing volunteers to gamma radiation from an external source; the authors do not provide [2] information about the degree of exposure to ionizing radiation that such method causes in

Pedro Berjano

pberjano@gmail.com

1 IRCCS Istituto Ortopedico Galeazzi, Via Riccardo Galeazzi, 4, 20161 Milan, Italy volunteers and such information is not readily available in the literature).

Second, the method to calculate the influence of arm position on the position of the body center of mass was deductive and not experimental.

Third, the mathematical model shows one over-simplification: just the anterior displacement of the olecranon has been utilized to estimate the arm position. The proposed formula would provide similar results for an upper limb position with $30^{\circ}$ of forward flexion of the arm and horizontal forearm as with $30^{\circ}$ of shoulder flexion and vertical forearm, despite the obvious difference in upper limb mass distribution between the two scenarios.

However, this is a relevant study. Its main strength is to provide a calculation method to estimate the influence of changes in arm position on the displacement of the body center of mass. Future investigations might validate or improve its findings.

Compliance with ethical standards

Conflict of interest I have no potential conflict of interest.

\section{References}

1. Legaye J, Duval-Beaupère G, Hecquet J et al (1998) Pelvic incidence: a fundamental pelvic parameter for three-dimensional regulation of spinal sagittal curves. Eur Spine J 7:99-103

2. Duval-Beaupère G, Schmidt C, Cosson P (1992) A barycentremetric study of the sagittal shape of spine and pelvis: the conditions required for an economic standing position. Ann Biomed Eng 20(4):451-462 